\title{
Structure, morphology and magnetism of an ultra-thin [NiO/CoO]/PtCo bilayer with perpendicular exchange bias
}

\author{
Helio C. N. Tolentino, Maurizio De Santis, Jean-Marc Tonnerre, Aline \\ Y. Ramos, Veronique Langlais, Stephane Grenier, and Aude Bailly \\ Institut Néel, UPR 2940, CNRS\&UJF, 25 Av. des Martyrs, BP 166, 38042 Grenoble, France*
}

(Received on 15 July, 2008)

\begin{abstract}
Electronic and magnetic properties of nanoscale materials are closely related to the atomic arrangement at the interface shared by different chemical elements. A very precise knowledge of the surface/interface structure is then essential to properly interpret the new properties coming out. Of a particular interest is the relationship between structure, morphology and magnetic properties of exchanged-coupled interfaces in ferromagnetic (FM) and antiferromagnetic (AF) materials. The interaction at the AF/FM interface modifies the magnetic switching properties of the FM film, which turn out to be a usefull property on new magnetic devices technology. We present here an investigation of the buried exchange-coupled interface $[\mathrm{NiO} / \mathrm{CoO}] /[\mathrm{PtCo}]$ grown on a $\mathrm{Pt}(111)$ single crystal. The magneto-optical Kerr effect reveals a strong coupling at the interface, by an increasing coercivity, and a spin reorientation of the FM film when ordering occurs in the AF layer. The combination of grazing incidence $\mathrm{X}$-ray diffraction, $\mathrm{X}$-ray reflectivity and soft $\mathrm{X}$-ray resonant magnetic scattering yields a comprehensive description of the system.
\end{abstract}

Keywords: magnetism, exchange bias, MOKE, synchrotron light.

\section{INTRODUCTION}

Reduced dimensionality and interface interaction are often at the origin of new properties in ultra-thin films, playing a crucial role in modern technologies. Twenty years ago, the association of ferromagnetic (FM) and non-ferromagnetic (NF) thin layers in a multilayered material lead to the discover of the giant magnetoresistance (GMR) effect [1]. This property represents an important breakthrough in science and opens the way for building up new sensors and magneto storage devices. GMR based exchange biased magnetic tunnel junctions (MTJ) and spin valves (SV) have useful properties for forming magnetic memory elements in novel device architecture [2]. The exchange bias (EB) effect [3] occurs when an antiferromagnetic (AF) material is placed in contact with a ferromagnetic (FM) one. The interaction at the AF/FM interface yields an increase of the magnetic field necessary for switching the magnetization and induces an unidirectional anisotropy. The role of the EB effect in devices is to magnetically pin one of the FM layers.

The EB effect was discovered half a century ago by Meiklejohn and Bean when studying magnetic properties of fine Co particles that turned out to be covered by a thin oxide layer $[3,4]$. About ten years ago, this phenomenon was revived and comprehensive reviews focused on many experimental $[5,6]$ and theoretical $[7,8]$ aspects were published. The basic description of the EB phenomenon was given since the pioneering work. For a temperature $\mathrm{T}$ above the AF ordering Nel temperature $\left(T_{N}\right)$ and below the FM layer Curie temperature $\left(T_{C}\right)$, the AF spins are disordered while the FM are ordered (Fig.1-a). A magnetic field $\mathrm{H}$ is applied in order to saturate the FM layer in a direction either parallel or perpendicular to the film surface. After cooling the AF/FM bilayer under such a magnetic field below $T_{N}$, the AF spins at the interface couple with the FM spins (Fig.1-b), yielding the energetically stable situation for the coupling at the interface. When the

*Electronic address: helio.tolentino@grenoble.cnrs.fr

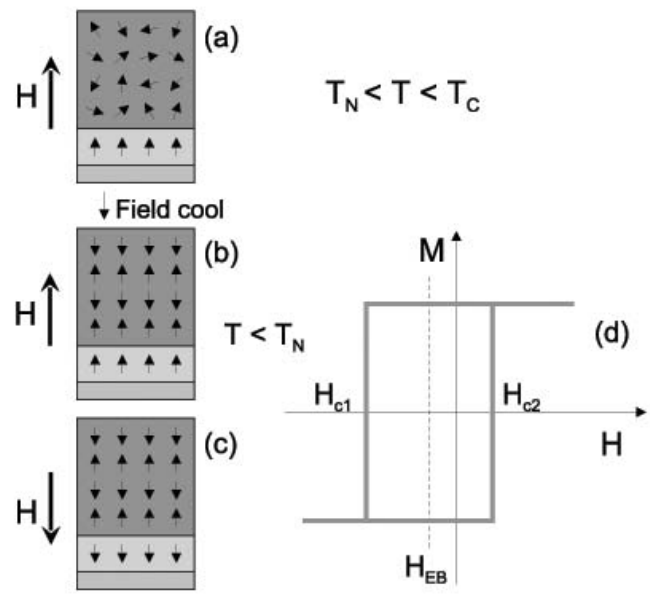

FIG. 1: Schematic illustration of the spin configuration of an AF/FM bilayer: a) above the AF ordering temperature $T_{N}$, when the $\mathrm{AF}$ spins are disordered; b) upon cooling under an applied magnetic field the AF spins close to the interface couples and align with the FM spins; c) the applied magnetic field is reversed: the FM spins follows the applied field while the AF spins are not directely affected; d) scheme of the exchange bias loop shift.

magnetic field $\mathrm{H}$ is reversed, the AF spins exert a microscopic torque on the FM spins, tending to keep them in their original direction (Fig.1-c). The field needed to reverse the magnetization will be larger $\left(\left|H_{C 1}\right|>\left|H_{C 2}\right|\right)$ and the magnetic loop will be shifted by an amount $H_{E B}=\left(H_{C 1}+H_{C 2}\right) / 2$, due to this additional interfacial magnetic energy, $\Delta \sigma$, that has to be overcome (Fig.1-d).

The phenomenological expression of the exchange field is $H_{E B}=\Delta \sigma /\left(M_{F M} \times t_{F M}\right)$, where $M_{F M}$ and $t_{F M}$ are the magnetic moment density and the thickness of the FM layer, respectively. While this basic description of the exchange bias phenomenon is generally accepted, the microscopic interfacial interactions that yields larger coercivity and exchange bias shift and contributes to the interfacial energy are more controversial. The calculated interfacial magnetic energy 
density, $\Delta \sigma$, exceeds the experimental values by orders of magnitude.

Many microscopic models have been proposed for the expression of the total magnetic energy and, in particular, for the interface exchange energy [9-14]. Among the complex phenomena taking place close to the interface, domain-wall formation in the AF layer, random interface roughness, contribution of compensated AF/FM interfaces, extension of the coupling beyond interfacial layers [15] and noncollinear interface spin configuration are to be considered. Moreover, the role of pinned and unpinned (AF spins that rotate) spins, or switchable interfacial uncompensated $\mathrm{AF}$ spins, in the exchange bias has been recently revealed by X-ray photoemission microscopy and X-ray magnetic linear and circular dichroism $[16,17]$. The study of ideal systems, with atomic scale control and fine characterization of the interface structure and morphology, is essential to disentangle all these parameters.

In AF materials the direction of the atomic moments varies on the length scale of nearest atomic distances. Recently, it has been shown that the magnetic coupling across the AF/FM interface in the $\mathrm{FeMn} / \mathrm{Co}$ system is mediated by step edges of single-atom height [18]. The authors showed that it is possible to tune the strength of the magnetic coupling among the FM layers across ultra-thin AF one and that the coupling is stronger if steps are distributed in small islands. The reason is that the coupling is mainly mediated by uncompensated spins at monoatomic step edges. This result demonstrates why roughness is so important in some EB systems. A quite different situation takes place at the $\mathrm{Fe} / \mathrm{NiO}(001)$ interface. The concomitant expansion of the interlayer distance and the small buckling of an interfacial $\mathrm{FeO}$ layer lead to an increase of the spin magnetic moment of the interfacial $\mathrm{Fe}$ atoms, which modifies dramatically the exchange interaction [19]. The expanded interlayer distance and buckling seems to be more important to the $\mathrm{Fe} / \mathrm{NiO}(001) \mathrm{AF} / \mathrm{FM}$ magnetic coupling than the presence of low density defective sites.

These two examples clearly demonstrate that complex surface interactions are the origin of EB. The electronic and magnetic properties of these nanoscale materials are closely related to the atomic arrangement at the interface shared by different chemical elements. It is then crucial to gather a very precise knowledge of the surface/interface structure in order to understand such new coming out properties. The availability of synchrotron sources lead to a wealth of well-established tools for structural analysis of surfaces, in particular grazing incidence X-ray diffraction, scattering and absorption spectroscopy techniques [20]. In addition, element-selective magnetic probes, as $\mathrm{x}$-ray magnetic circular dichroism (XMCD) and $\mathrm{x}$-ray resonant magnetic scattering (XRMS), became available and complement structural and other conventional magnetic probes. Since the AF/FM interface is buried and changes in the structural and magnetic properties are small, combining all these techniques is of paramount importance to tackle the challenging description of such systems.

The major part of exchange bias studies have been performed with the magnetization parallel to the FM/AF interface. Studies on systems with perpendicular (out of plane) magnetic anisotropy (PMA) are rather recent [21-23] and only few address the role of spin configuration at the interface $[24,25]$. We are especially interested in thin ferromagnetic films with PMA, as is the case in FePt and CoPt surface alloys, coupled to antiferromagnets, like $\mathrm{CoO} / \mathrm{NiO}$ mixed oxides. PMA is recognized as a way for increasing magnetic storage density. In addition, magneto optical effects are enhancend at polar (sensitive to perpendicular magnetization component) geometry compared to in-plane one [26, 27].

We report here on a combined structural and magnetic study of the $[\mathrm{NiO} / \mathrm{CoO}] /[\mathrm{PtCo}]$ perpendicular exchange bias system. Previous experiments on a sputtered $\mathrm{Co} / \mathrm{Pt}$ multilayer with PMA coupled to a $\mathrm{CoO}$ oxide showed that such a system exhibits loop shifts and enhanced coercivities for both parallel and perpendicular applied magnetic fields [22]. The aim of our investigation is to obtain a fine control of the structure and to reach a comprehension of the exchange coupling mechanism at the interface of $\mathrm{AF} / \mathrm{FM}$ systems that are as close as possible to model ones. Our samples have been prepared by electron beam epitaxy and oxidized in a controlled oxygen partial pressure, as described in the next section. $\mathrm{CoO}$ is an AF oxide with $T_{N}=293 \mathrm{~K}$ and a large magnetocrystalline anisotropy. $\mathrm{NiO}$ presents a much higher ordering temperature, $T_{N}=523 \mathrm{~K}$, and smaller anisotropy. By mixing both oxides we obtained an AF material with a high magnetocrystalline anisotropy and with a Nel temperature intermediate between those of the two oxides [28]. The combination of grazing incidence $\mathrm{X}$-ray diffraction, $\mathrm{X}$-ray reflectivity and soft X-ray resonant magnetic scattering yields a comprehensive description of the system.

\section{RESULTS AND DISCUSSION}

\section{A. Sample preparation, structure and morphology}

An epitaxial [NiO(3ML)/CoO(3ML)]x3/PtCo sample was synthesized by sequential electron beam evaporation over a $\mathrm{Pt}(111)$ single crystal in the ultra-high vacuum (UHV) chamber of the French CRG BM32 beamline at ESRF [29]. The layer by layer growth described hereafter was followed step by step by in situ grazing incidence $\mathrm{X}$-ray diffraction $[30,31]$. The structural characteristic of the sample is then perfectly known at each stage of the growth. The very stable oxide layer on top surface enables posterior ex-situ structural, morphological and magnetic studies.

One monolayer (1 ML) of Co was deposited onto a $\mathrm{Pt}(111)$ single crystal held at $540 \mathrm{~K}$, previously cleaned under UHV following standard procedures [32]. These conditions promote the formation of a PtCo surface alloy with about $90 \%$ of the Co atoms buried under a Pt layer that terminates the surface. Such a PtCo surface alloy is ferromagnetic (FM) and displays a strong perpendicular magnetic anisotropy (PMA). A coercivity of $1.2 \mathrm{kOe}$ was observed in situ by XMCD at the Co L edges in a similar system [33]. This PtCo(111) surface alloy was then held at $450 \mathrm{~K}$ during the procedure of [NiO/CoO] multilayer deposition, performed as follows. 1 ML of Co was deposited and then oxidized by exposure to 50 Langmuir of oxygen. This procedure was repeated three times yielding an epitaxial $3 \mathrm{ML} \mathrm{CoO(111)} \mathrm{film} \mathrm{with} \mathrm{in-plane}$ bulk lattice constant. On top of it, a $3 \mathrm{ML} \mathrm{NiO(111)} \mathrm{film} \mathrm{was}$ grown following the same procedure. Such $\mathrm{NiO} / \mathrm{CoO}$ bilayer sequence was repeated three times, leading to the AF film of about $18 \mathrm{ML}$, schematically represented in figure 2 . 


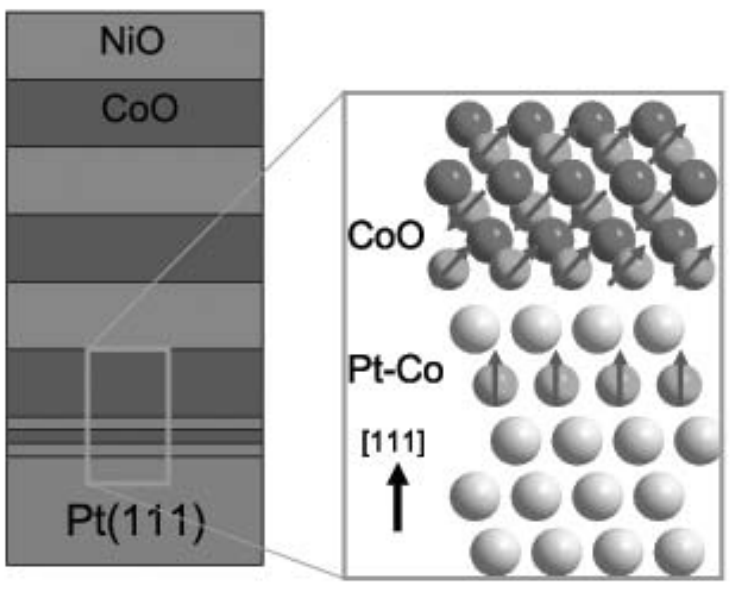

FIG. 2: Schematic sequence of the AF/FM bilayer $[\mathrm{NiO}(3 \mathrm{ML}) / \mathrm{CoO}(3 \mathrm{ML})] \times 3 / \mathrm{PtCo}$ sample. Insert: close view of the spin configuration at the interface $\mathrm{CoO} / \mathrm{PtCo}$, assuming that both layers are ordered and non-interacting

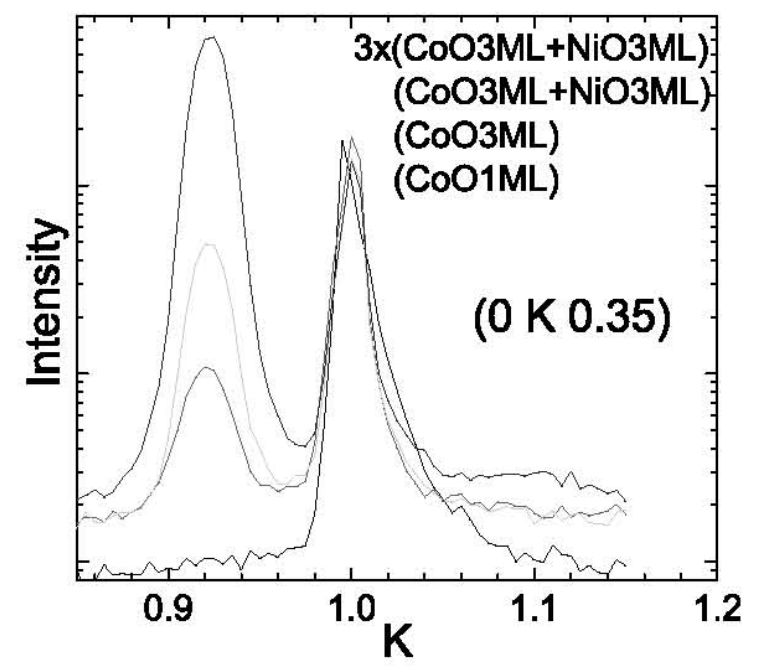

FIG. 3: Surface X-ray diffraction pattern during the growth of the $\mathrm{NiO} / \mathrm{CoO}$ mixed oxide over the $\mathrm{PtCo}(111)$ surface alloy

The mixed oxide grow in orientational epitaxy on the $\mathrm{Pt}(111)$ substrate (Fig. 3). The first $\mathrm{CoO}$ atomic layer is pseudomorphe on the substrate. After the second oxide layer deposition, the layer relaxes and a bulk-like $\mathrm{CoO}(111)$ lattice peak shows up at $\mathrm{K}=0.92$. An hexagonal unit cell was taken for the $\operatorname{Pt}(111)$ crystal $[32,34]$. The peak at $\mathrm{K}=1$ in the reciprocal space correspond to the interlayer spacing of 0.2266 $\mathrm{nm}$ for $\mathrm{Pt}$, yielding an oxide layer in-plane interlayer spacing of $0.245 \mathrm{~nm}$, exactly as in the $\mathrm{CoO}$ bulk oxide. The $\mathrm{NiO}$ layer grows in coherent epitaxy with the $\mathrm{CoO}$ and displays the same in-plane parameter, as observed by its contribution to diffraction peak intensity, at exactly the same position in the reciprocal space.

A well defined atomic stacking is observed at each step of the oxide deposition, as well as in the final sample. On the other hand, $\mathrm{CoO}$ diffraction rods as well posteriory ex situ measurements show that this final sample has a quite large

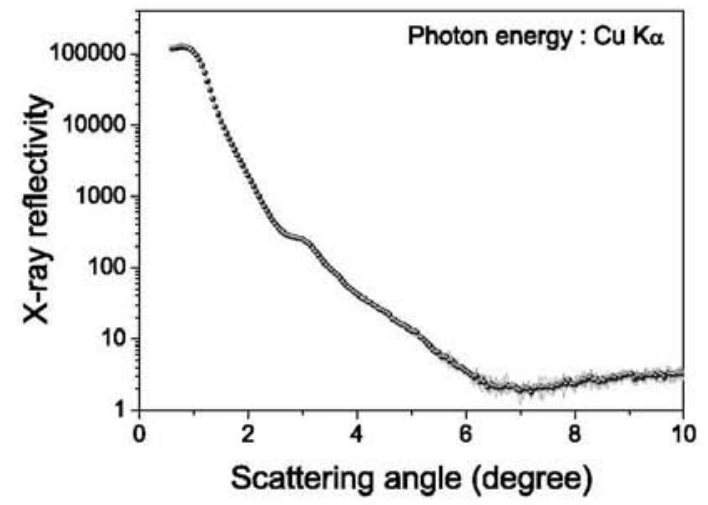

FIG. 4: $\mathrm{X}$ ray reflectivity $\left(\mathrm{Cu} K_{\alpha}, \lambda=0.154 \mathrm{~nm}\right.$ ) measurements from the rough oxide surface. The surface has a large roughness, $1 \mathrm{~nm}$, while the interface is quite flat, with roughness of about $0.1 \mathrm{~nm}$.

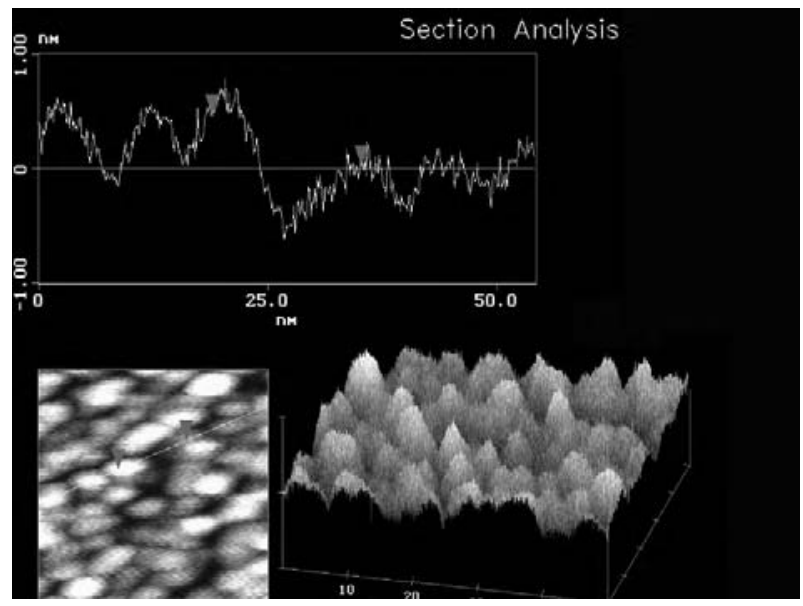

FIG. 5: AFM of a 12 ML CoO layer grown by electron beam evaporation on top of a $\operatorname{Pt}(111)$ single crystal. A roughness close to $1 \mathrm{~nm}$ can be observed

rough surface. Kiessig interference fringes [20] can hardly be observed in the X-ray reflectivity profile (Fig.4). The fitting to the calculated reflectivity gives a roughness of about $1 \mathrm{~nm}$ at the surface, while the interface is rather flat, with roughness of about $0.1 \mathrm{~nm}$. Such a large roughness is not surprinsing due to the polar character of the $\operatorname{Pt}(111)$ surface. AFM measurements in a similar oxide surface - a $12 \mathrm{ML} \mathrm{CoO} \mathrm{film} \mathrm{on}$ the same $\operatorname{Pt}(111)$ crystal surface, treated in identical conditions - also reveal that the surface roughness is roughly $1 \mathrm{~nm}$ (Fig.5).

As a matter of fact, owing to their sligthly different inplane lattice parameters, the superposition of the $\mathrm{CoO}(111)$ and $\operatorname{Pt}(111)$ lattices gives rise to a Moire structure with a 3.6 $\mathrm{nm}$ periodicity. Such Moire modulation, originates from the coincidence of $12 \mathrm{CoO}$ units with $13 \mathrm{Pt}$ atomic distances and might be used as a template for nucleating nanoparticles, as in a similar $\mathrm{FeO} / \mathrm{Pt}(111)$ surface [35]. The combination of epitaxy, stacking and roughness could be well explained by a preferential nucleation of $\mathrm{CoO}$ islands on such a Moire structure. 


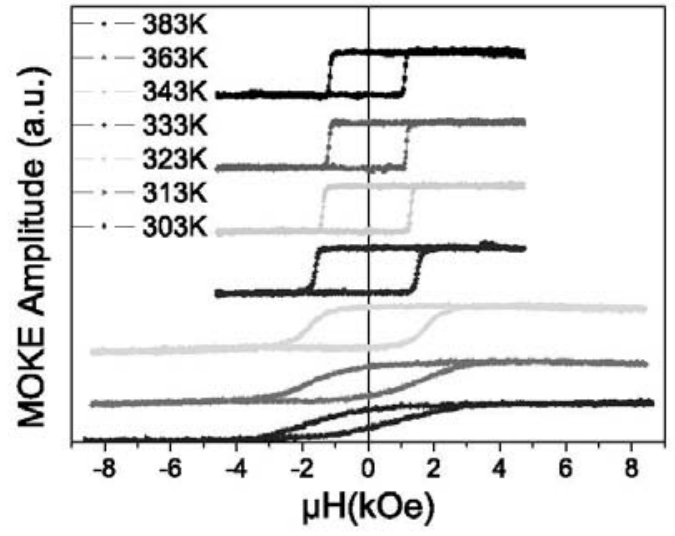

FIG. 6: PMOKE hysteresis loops measured at different temperatures, after field cooling from $400 \mathrm{~K}$ under $5 \mathrm{kOe}$.

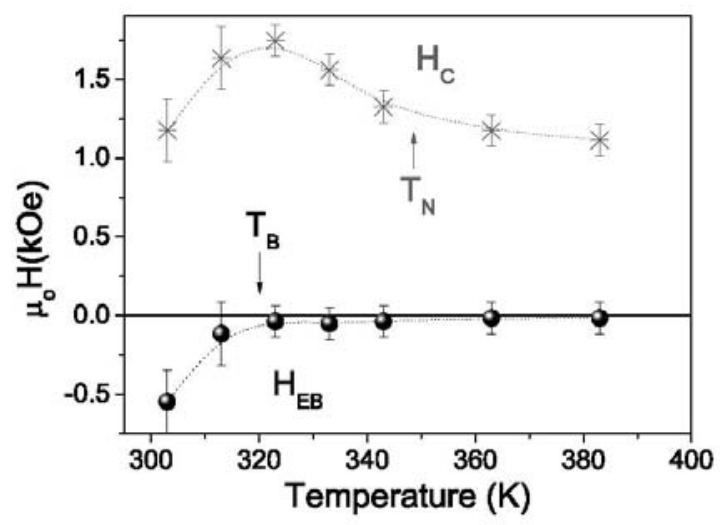

FIG. 7: Exchange bias shift (circles-black) and coercivity (stars-red) as function of temperature. The blocking temperature $T_{B}$ is estimated at $320 \mathrm{~K}$, close to the maximum coercivity.

\section{B. Increased coercivity, exchange bias and spin reorientation}

Magneto-optic Kerr effect (MOKE) was used to follow the magnetic properties of our sample. MOKE is a wellestablished technique to study magnetism in ultra-thin FM films [26]. The MOKE sub-monolayer sensitivity has been verified in many situations [27]. Polar and longitudinal MOKE are characterized by a complex rotation of the plane of polarization of the linearly polarized incident light upon reflection from the surface of a ferromagnetic material. The rotation is directly related to the magnetization of the material within the probed region of the light.

In order to induce EB, the sample was field cooled from above the Nel temperature down to room temperature under an applied magnetic field of $5 \mathrm{kOe}$. Then, polar MOKE (PMOKE) hysteresis loops were measured at increasing temperatures, up to $383 \mathrm{~K}$ (Fig. 6). The coercivity $\left(H_{C}=\right.$ $\left(H_{C 2}-H_{C 1}\right) / 2$ ) presents a maximum of $1.7 \mathrm{kOe}$ at about 320 $\mathrm{K}$, then decreases monotonically to $1.2 \mathrm{kOe}$ at higher temperatures (Fig.7). It is worth noting that $1.2 \mathrm{kOe}$ is the same value as for the PtCo surface alloy without any capping oxyde [33]. The FM layer preserves its quality and is altered just by the exchange coupling to the $\mathrm{AF}$ ordered layer.

For temperatures below $320 \mathrm{~K}$ the hysteresis loops shifts. The temperature below which the shift appears is defined as the blocking temperature, $T_{B}=320 \mathrm{~K}$. At room temperature, the shift is found to be $H_{E B}=-0.6 \mathrm{kOe}$ and characterises the perpendicular exchange coupling at the interface between the FM and AF layers. From that, the calculated interfacial exchange energy is $\Delta \sigma=0.16 \mathrm{erg} / \mathrm{cm}^{2}$, where we used the FM thickness as $t_{F M}=0.36 \mathrm{~nm}$. This is about $2 / 3$ of the value found by Maat et al. [22] for a multilayer at $10 \mathrm{~K}$. The most striking result in this range is that the hysteresis loops becomes less and less squared, as indicated by the decreasing PMOKE amplitude at remanence and loop elongation (Fig. 6 and 7). The decreasing squareness of the loops indicates that the easy magnetization axis of the FM layer is no longer perpendicular. Our interpretation is that, upon field cooling, the AF spins should align along the spin anisotropy axis that is closest to the applied magnetic field [36]. This should be particularly applicable for $\mathrm{CoO}$ because of its high magnetocrystalline anisotropy constant around the $\{117\}$ directions. The $\mathrm{CoO}$ spins will be oriented along one of the $\{117\}$ directions, forming an angle of 43.3 with the surface normal. The orientation of the $\mathrm{NiO}$ magnetic moments is assumed to follow the $\mathrm{CoO}$ spins because of a strong exchange interaction at the interface [37] and a smaller magnetocrystalline anisotropy constant. Therefore, the change in the hysteresis loops is related to the reorientation of the Co spins in the FM layer due to exchange coupling with the oxide layer. As far as we know, this is the first experimental observation of the reorientation of the out-of-plane interfacial FM spins induced by the ordering of the AF layer. One should note the fact that, in other $[\mathrm{Co} / \mathrm{Pt}] /[\mathrm{CoO}]$ systems, the FM layer is thicker than a single monolayer (e.g. Maat et al [22]).

For temperatures above $320 \mathrm{~K}$ the hysteresis loops are squared, with a magnetization at remanence close to magnetization at saturation. In this temperature range there is no exchange shift. The increased coercivity $H_{C}$ is related to the antiferromagnetic order persisting in the mixed oxide layer. The AF ordering temperature $T_{N}$ may be estimated by the inflexion point of the coercivity as function of temperature [38] and turns out to be $T_{N}=350 \mathrm{~K}$. This value is smaller by $50 \mathrm{~K}$ compared to the average $\mathrm{CoO}$ and $\mathrm{NiO}$ bulk Nel temperatures due to the reduced thickness effect which lowers the order temperature [36]. As can be also observed, $T_{B}$ is smaller by $30 \mathrm{~K}$ than $T_{N}$, owing to thermal activation of the AF domains. Such kind of difference has already been pointed out by Maat et al. for the pure $\mathrm{CoO}$ coupled to a $\mathrm{Co} / \mathrm{Pt}$ multilayer.

\section{Depth profile spin configuration at the interface}

The depth dependence of the out-of-plane magnetization across the interface between the AF and FM layer has been investigated taking advantage of the chemical and spatial sensitivity of soft X-ray Magnetic Reflectivity [39]. This polarized synchrotron X-ray technique exploits the magnetization sensitivity of the atomic scattering factor (ASF) at an absorption edge and is known to be sensitive to the three directions of the magnetization with spatial, chemical and orbital selectivity [40]. The soft $\mathrm{x}$-ray specular reflectivity experiments were conducted on the SIM beamline at the Swiss Light Source us- 


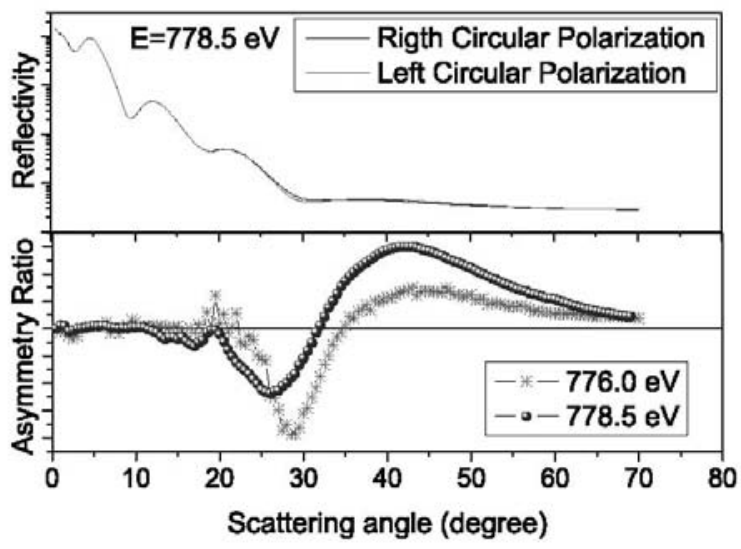

FIG. 8: XRMS at the Co $L_{3}$ edge. Upper curves correspond to the two polarizations at $E=778.5$. The curves display the asymmetry ratio at two different energies about the edge.

ing the RESOXS endstation [41]. The magnetic saturation of the FM layer was achieved by a 4 kOe permanent magnet brought to the sample perpendicularly to its surface.

The measurements were carried out in remanence at $\mathrm{T}=$ $340 \mathrm{~K}$, right above $T_{B}$ and below $T_{N}$, where the FM layer has a strong PMA and where the interfacial magnetic coupling with the AF layer constrains the reversal process, as indicated by the increased $H_{C}$ (Fig. 7). The reflected intensity was recorded as a function of the scattering angle at different photon energies, and as function of energy at fixed scattering settings. The asymmetry ratios, or normalized dichroic differences, were measured using $98 \%$ left and right circularly polarized beam [42]. Absorption spectra were collected simultaneously by recording the drain current as a function of incident photon energy. The spectral shape of the absorption is essentially that of the $\mathrm{CoO}$ and $\mathrm{NiO}$ oxide, as expected from a total electron yield measurement[43]. In reflectivity condition, the penetration depth is large enough to probe the buried Co layer.

Angle dependent reflected intensity (Fig. 8-a) were collected at 776.9 and $778.5 \mathrm{eV}$ close to the $\mathrm{Co} L_{3}$ edge. At both energies, a separation of the curves with right and left polarized ligth is observed. The asymmetry ratios at both energies are close to zero at small angles and exhibit a larger amplitude at high angles (Fig. 8-b), in agreement with the geometrical dependence of the atomic scattering factor arising from an out-of-plane magnetization component. This angle dependence is mainly due to the Co magnetization in the PtCo layer and in the oxide layer.

The structural parameters of the film were derived from the the refinement of the average reflectivity. The total thicknesses is about $5.45 \mathrm{~nm}$ of oxide and $0.36 \mathrm{~nm}$ of PtCo. This is in good agreement with the expected thickness from the sequential deposition of $18 \mathrm{ML}$ of oxide and $1 \mathrm{ML}$ of Co. The roughnesses are 0.02 and $0.9 \mathrm{~nm}$ for the interface $\mathrm{CoO} / \mathrm{PtCo}$ and for the top $\mathrm{NiO} / \mathrm{CoO}$ interfaces, respectively, is in agreement the X-ray reflectivity and AFM results, too.

The magnetic profile comes from the analysis of the asymmetry ratio at both energies. The interference at about 32 indicates a magnetic thickness smaller than the total oxide thickness. A model assuming that $\mathrm{Co}$ atoms in the oxide may be magnetically ordered up to the first $\mathrm{CoO} / \mathrm{NiO}$ interface works well. The refinement of the magnetic structure was performed by dividing the first $\mathrm{CoO}$ layer in three slices and by adjusting their thicknesses as well as the magnetic moments carried by the Co atoms. The results indicate the out-of-plane magnetization is distributed beyond the $0.36 \mathrm{~nm}$ Pt-Co layer and extends over $1 \mathrm{~nm}$ in the oxide layer. The coupling to the FM Co spins is parallel in a $0.3 \mathrm{~nm}$ thick slice, roughly the first $\mathrm{CoO}$ monolayer.Then, it is antiparallel in a $0.7 \mathrm{~nm}$ thick one, with similar amplitude. The last slice that completes the $\mathrm{CoO}$ layer is found to be $0.2 \mathrm{~nm}$ thick and has no net magnetization. Considering models with no magnetization in the oxide layer, completely parallel or antiparallel magnetic slices and more extended magnetization with reduced magnetic amplitude do not fit the interference effect observed experimentally. This result can be understood as follows: the Co atoms in the first $\mathrm{CoO}$ slice, right on top of the Pt-Co layer, have a mixed electronic character in between metallic and oxidized state. Their net induced magnetization are likely to be dominated by the proximity of the Pt-Co layer and are ferromagnetically coupled to it. For the next $\mathrm{CoO}$ slice, the $\mathrm{Co}$ atoms are fully oxidized and the net magnetization is antiferromagnetically coupled to the first one. The net magnetization found equal to zero in the third slice means that beyond the second oxide layer the AF material break into domains that are not biased by the field cooling processes.

\section{SUMMARY}

Our results show that valuable insights into the surface structure and magnetism in the $\mathrm{CoO} / \mathrm{PtCo}$ system emerge from combination of in situ, ex situ, and in-depth sensitive structural and magnetic characterizations. In situ grazing incidence X-ray diffraction (GIXRD) results demonstrate that the AF oxide grows epitaxially on top of the PtCo(111) surface alloy. The top surface of the mixed oxide is rough while the interface $\mathrm{CoO} / \mathrm{PtCo}$ is quite flat over the whole crystal. The perpendicular magnetic anisotropy, well above the $\mathrm{Nel}$ temperature, is the same as for the uncapped PtCo layer, showing that the quality of the layer is preserved after oxide deposition. Upon field cooling, slightly above $T_{B}$, the ultra thin Co ferromagnetic layer exhibits a strong perpendicular anisotropy and magnetic ordering is induced over a few oxide atomic layers. The out-of-plane magnetic profile shows that oxidized Co atoms closest to the interface are ferromagnetically coupled to the PtCo ferromagnetic layer. The second oxide layer then couples to this interfacial one antiferromagnetically. Below the blocking temperature $T_{B}$, MOKE indicates a spin reorientation in the FM layer that would follow the blocked Co spins of the AF layers. Such reorientation in that FM surface layer indicates that the interfacial spins in a thicker FM layer is strongly modified by the ordering of the top AF layer.

\section{Aknowledgements}

We would to acknowledge the French CRG BM32 beamline at ESRF and the SIM beamline at SLS, specially U. Staub for his help and discussions. Yves Souche (Institut 
[1] M. N. Baibich, J. M. Broto, A. Fert, F. N. Van Dau, F. Petroff, P. Eitenne, G. Creuzet, A. Friederich, and J. Chazelas, Phys. Rev. Lett. 61, 2472 (1988).

[2] S. Parkin, K. Roche, M. Samant, P. Rice, R. Beyers, R. Scheuerlein, E. OSullivan, S. Brown, J. Bucchigano, D. Abraham, et al., J. Appl. Phys. 85, 5828 (1999).

[3] W. H. Meiklejohn and C. P. Bean, Phys. Rev. 102, 1413 (1956).

[4] W. H. Meiklejohn and C. P. Bean, Phys. Rev. 105, 904 (1957).

[5] J. Nogues and I. K. Schuller, J. Magn. Magn. Mater. 192, 203 (1999).

[6] A. E. Berkowitz and K. Takano, J. Magn. Magn. Mater. 200, 552 (1999).

[7] M. Kiwi, J.Mag.Mag.Mat. 234, 584 (2001).

[8] R. Stamps, J.Phys.D:Appl.Phys. 33, R247 (2000).

[9] D. Mauri, H. Siegmann, P. Bagus, and E. Kay, J. Appl. Phys. 62, 3047 (1987)

[10] A. P. Malozemoff, Phys. Rev. B 35, 3679 (1987).

[11] N. C. Koon, Phys. Rev. Lett. 78, 4865 (1997).

[12] M. D. Stiles and R. D. McMichael, Phys. Rev. B 59, 3722 (1999).

[13] U. Nowak, K. D. Usadel, J. Keller, P. Miltényi, B. Beschoten, and G. Güntherodt, Phys. Rev. B 66, 014430 (2002).

[14] K. Lee, Y. Yu, and S. Kim, Appl. Phys. Lett. 86, 192512 (2005).

[15] J. Kortright, D. Awschalom, J. Stohr, S. Bader, Y. Idzerda, S. Parkin, I. Schuller, and H. Siegmann, J.Mag.Mag.Mat. 207, 7 (1999).

[16] H. Ohldag, T. J. Regan, J. Stöhr, A. Scholl, F. Nolting, J. Lüning, C. Stamm, S. Anders, and R. L. White, Phys. Rev. Lett. 87, 247201 (2001).

[17] H. Ohldag, A. Scholl, F. Nolting, E. Arenholz, S. Maat, A. T. Young, M. Carey, and J. Stöhr, Phys. Rev. Lett. 91, 017203 (2003).

[18] W. Kuch, L. Chelaru, F. Offi, J. Wang, M. Kotsugi, and J. Kirschner, Nature Materials 5, 128 (2006).

[19] P. Luches, V. Bellini, S. Colonna, L. D. Giustino, F. Manghi, S. Valeri, and F. Boscherini, Physical Review Letters 96, 106106 (pages 4) (2006).

[20] A. Nielsen, An Introduction to X-Ray Physics (Elsevier Science, 2000).

[21] B. Kagerer, C. Binek, and W. Kleemann, J. Magn. Magn. Mater. 217, 139 (2000).

[22] S. Maat, K. Takano, S. S. P. Parkin, and E. E. Fullerton, Phys. Rev. Lett. 87, 087202 (2001).

[23] J. Sort, V. Baltz, F. Garcia, B. Rodmacq, and B. Dieny, Physical Review B (Condensed Matter and Materials Physics) 71, 054411 (pages 7) (2005).
[24] S. M. Zhou, L. Sun, P. C. Searson, and C. L. Chien, Phys. Rev. B 69, 024408 (pages 5) (2004).

[25] A. Baruth, D. J. Keavney, J. D. Burton, K. Janicka, E. Y. Tsymbal, L. Yuan, S. H. Liou, and S. Adenwalla, Phys. Rev. B 74, 054419 (pages 13) (2006).

[26] S. Bader, J.Mag.Mag.Mat. 100, 440 (1991).

[27] Z. Qiu and S. Bader, J.Mag.Mag.Mat. 200, 664 (1999).

[28] J. A. Borchers, M. J. Carey, R. W. Erwin, C. F. Majkrzak, and A. E. Berkowitz, Phys. Rev. Lett. 70, 1878 (1993).

[29] R. Baudoing-Savois, M. De Santis, M. Saint-Lager, P. Dolle, O. Geaymond, P. Taunier, P. Jeantet, J. Roux, G. Renaud, A. Barbier, et al., Nucl. Instrum. and Meth. in Phys. Res. B 149, 213 (1999).

[30] E. Vlieg, J. F. Van Der Veen, S. J. Gurman, C. Norris, and J. E. Macdonald, Surface Science 210, 301 (1989).

[31] I. K. Robinson, Handbook of Synchrotron Radiation, vol. 3 (North Holland - Amsterdam, 1991).

[32] M. De Santis, R. Baudoing-Savois, P. Dolle, and M. C. SaintLager, Phys. Rev. B 66, 085412 (2002).

[33] L. Giovanelli, M. De Santis, G. Panaccione, F. Sirotti, P. Torelli, I. Vobornik, R. Larcipretea, S. Egger, M. SaintLager, P. Dolle, et al., J. Magn. Magn. Mater. 288, 236 (2005).

[34] B. E. Waren, X-Ray Diffraction (Dover Publications - New York, 1994).

[35] N. Berdunov, G. Mariotto, K. Balakrishnan, S. Murphy, and I. V. Shvets, Surface Science 600, L287 (2006).

[36] D. Alders, L. H. Tjeng, F. C. Voogt, T. Hibma, G. A. Sawatzky, C. T. Chen, J. Vogel, M. Sacchi, and S. Iacobucci, Phys. Rev. B 57, 11623 (1998).

[37] M. J. Carey and A. E. Berkowitz (AIP, 1993), vol. 73, pp. 6892-6897.

[38] K. Lenz, S. Zander, and W. Kuch, Physical Review Letters 98 , 237201 (pages 4) (2007)

[39] J. M. Tonnerre, L. Sève, D. Raoux, G. Soullié, B. Rodmacq, and P. Wolfers, Phys. Rev. Lett. 75, 740 (1995).

[40] J. P. Hill and D. F. McMorrow, Acta Crystallographica Section A 52, 236 (1996).

[41] N. Jaouen, J.-M. Tonnerre, G. Kapoujian, P. Taunier, J.-P. Roux, D. Raoux, and F. Sirotti, Journal of Synchrotron Radiation 11, 353 (2004).

[42] J. M. Tonnerre, M. D. Santis, S. Grenier, H. C. N. Tolentino, V. Langlais, E. Bontempi, M. García-Fernández, and U. Staub, Physical Review Letters 100, 157202 (pages 4) (2008).

[43] T. J. Regan, H. Ohldag, C. Stamm, F. Nolting, J. Lüning, J. Stöhr, and R. L. White, Phys. Rev. B 64, 214422 (2001). 